\title{
Power domination in regular claw-free graphs *
}

\author{
Changhong Lu Rui Mao Bing Wang \\ Department of Mathematics, \\ Shanghai Key Laboratory of PMMP, \\ East China Normal University, \\ Shanghai 200241, P. R. China \\ C. Lu email: chlu@math.ecnu.edu.cn \\ R. Mao(凶) email: maorui1111@163.com \\ B. Wang email: wuyuwuyou@126.com
}

June 9, 2021

\begin{abstract}
In this paper, we first show that the power domination number of a connected 4-regular claw-free graph on $n$ vertices is at most $\frac{n+1}{5}$, and the bound is sharp. The statement partly disprove the conjecture presented by Dorbec et al. in SIAM J. Discrete Math., 27:1559-1574, 2013. Then we present a dynamic programming style linear-time algorithm for weighted power domination problem in trees.
\end{abstract}

Keywords. Power domination, claw-free graph, regular, weighted tree

\section{Introduction}

Electric power systems need to be continually monitored. One way to fulfill this task is to place phase measurement units at selected locations in the system. The power system monitoring problem, as introduced in [2], asks for as few as possible measurement devices to be put in an electric power system. The power system monitoring problem was then described as a graph theoretical problem in [13]. The problem is

*Supported in part by National Natural Science Foundation of China (Nos. 11371008 and 91230201). 
similar to a problem of domination, in which, additionally, the possibility of some propagation according to Kirschoff laws is considered.

Let $G=(V, E)$ be a connected, simple graph with vertex set $V=V(G)$ and edge set $E=E(G)$. The open neighborhood of a vertex $v \in V(G)$ is the set $N_{G}(v)=\{u \in$ $V(G) \mid u v \in E(G)\}$, and the degree of $v$ is $d_{G}(v)=\left|N_{G}(v)\right|$. The closed neighborhood of $v$ is the set $N_{G}[v]=N_{G}(v) \cup\{v\}$. A graph $G$ is $k$-regular if $d_{G}(v)=k$ for every vertex $v \in V(G)$. The open neighborhood of a subset $S \subseteq V$ of vertices is the set $N_{G}(S)=\cup_{v \in S} N(v)$, while the closed neighborhood of $S$ is the set $N_{G}[S]=N_{G}(S) \cup S$. We denote $K_{i, j}$ the complete bipartite graph with two partite sets of cardinality $i$ and $j$, respectively. A claw-free graph is a graph that does not contain a claw, i.e., $K_{1,3}$, as an induced subgraph. We say a subset of $V(G)$ an independent set if no two vertices of the set are adjacent in $G$. Let $x$ and $y$ are two vertices of $G$. Denote by $d(x, y)$ the distance of $x$ and $y$ in $G$. We say a subset of $V(G)$ a packing if no two vertices in the set of distance less than three in $G$. For two graphs $G=(V, E)$ and $G^{\prime}=\left(V^{\prime}, E^{\prime}\right)$, let $G \cup G^{\prime}=\left(V \cup V^{\prime}, E \cup E^{\prime}\right)$ and $G \cap G^{\prime}=\left(V \cap V^{\prime}, E \cap E^{\prime}\right)$. If $G \cup G^{\prime}=\emptyset$, then $G$ and $G^{\prime}$ are disjoint. For any vertex subset $X$ of $G$, let $G-X=G[V \backslash X]$ and for $X=\{x\}$ let $G-x=G-\{x\}$ for short. For notation and graph theory terminology not defined herein, we in general follow [7].

The original definition of power domination was simplified to the following definition independently in [9, 10, 12, 17] and elsewhere.

Definition 1.1 Let $G$ be a graph. A set $S \subseteq V(G)$ is a power dominating set (abbreviated as $P D S$ ) of $G$ if and only if all vertices of $V(G)$ have messages either by Observation Rule 1 (abbreviated as OR 1) initially or by Observation Rule 2 (abbreviated as $O R$ 2) recursively.

OR 1. A vertex $v \in S$ sends a message to itself and all its neighbors. We say that $v$ observes itself and all its neighbors.

OR 2. If an observed vertex $v$ has only one unobserved neighbor $u$, then $v$ will send a message to $u$. We say that $v$ observes $u$.

Let $G=(V, E)$ be a graph and $S$ be a subset of $V$. For $i \geq 0$, we define the set $P_{G}^{i}(S)$ of vertices observed by $S$ at step $i$ by the following rules: 
(1) $P_{G}^{0}(S)=N[S]$;

(2) $P_{G}^{i+1}(S)=\cup\left\{N_{G}[v]: v \in P_{G}^{i}(S)\right.$ such that $\left.\left|N_{G}[v] \backslash P_{G}^{i}(S)\right| \leq 1\right\}$.

Note that if $S$ is a power domination set of $G$, then there is a minimal integer $i_{0}$ such that $P_{G}^{i_{0}}(S)=V(G)$. Hence $P_{G}^{j}(S)=P_{G}^{i_{0}}(S)$ for every $j \geq i_{0}$ and we accordingly define $P_{G}^{\infty}(S)=P_{G}^{i_{0}}(S)$. If the graph $G$ is clear from the context, we will remove the subscripts $G$ for short.

The power domination number of a graph $G$, denoted by $\gamma_{p}(G)$, is the minimum cardinality of a PDS of $G$. A PDS of $G$ with minimum cardinality is called a $\gamma_{p}(G)$ set. The power domination problem was known to be NP-complete even for bipartite graphs, planar graphs and split graphs, see [12, 13].

Chang et al [6] generalized the power domination to $k$-power domination by replacing the OR 2 with the following observation rule: If an observed vertex $v$ has at most $k$ unobserved neighbors, then $v$ will send a message to all its unobserved neighbors. The definition also can be found in [21].

The $k$-power domination number of $G$, denoted by $\gamma_{p, k}(G)$, is the minimum cardinality of a $k$-power dominating set of $G$. When $k=1$, The $k$-power domination is usual power domination. Both power domination and $k$-power domination are now well-studied in the literature (see, for example, [1, 6, 8, 9, 10, 12, 21, 23, 25]).

The paper is organized as follows. In section 2, we will give sharp upper bounds for the power domination number of a connected 4-regular claw-free graph on $n$ vertices. The result will partly disprove the conjecture introduced by Dorbec et al. in [8]. In section 3, we give a dynamic programming style linear-time algorithm for weighted power domination in trees.

\section{Power domination in 4-regular claw-free graphs}

Zhao et al. 25. proved that if $G$ is a connected claw-free cubic graph of order $n$, then $\gamma_{p}(G) \leq \frac{n}{4}$. Chang et al. [6] showed that if $G$ is a connected claw-free $(k+2)$ regular graph on $n$ vertices, then $\gamma_{p, k}(G) \leq \frac{n}{k+3}$. Recently, Dorbec et al. [8] gave an found that the claw-free condition can be removed. Then they presented the following conjecture. 
Conjecture 2.1 ([8]) For $k \geq 1$ and $r \geq 3$, if $G \not K_{r, r}$ is a connected r-regular graph of order $n$, then $\gamma_{p, k}(G) \leq \frac{n}{r+1}$.

It is obvious that if the conjecture holds for $k=1$, then it also holds for all $k \geq 2$. Hence, we pay our attention to the case of $k=1$ in the context. Dorbec et al. [8] showed that $\gamma_{p, k}(G) \leq \frac{n}{k+3}$ for any connected $(k+2)$-regular graph $G$ on $n$ vertices. It means that Conjecture 2.1 holds for $k=1$ and $r=3$. However, for each even $r \geq 4$, we show that Conjecture 2.1 does not always hold.

We first give a counterexample $E_{0}$ with $2 r+1$ vertices. Then based on $E_{0}$, we obtain infinitely many counterexamples of Conjecture 2.1 (in fact, the graphs are extremal graphs of Theorem 2.2 as well). Pick first two copies of $K_{r}$ and one singleton vertex $u$, then add $r$ independent edges between the two copies and $r$ edges linking the vertex $u$ to these vertices which are not incident to any independent edge before. Denote by the resulting $r$-regular graph $E_{0}$. Let $E_{1}$ be the graph obtained from $E_{0}$ by splitting the vertex $u$ into two vertices of degree $\frac{r}{2}$, say $u_{1}^{1}$ and $u_{2}^{1}$, then add an extra $K_{r}$ and link exactly $\frac{r}{2}$ edges from each $u_{i}^{1}$ to the new $K_{r}$ such that the resulting graph is $r$-regular as well. Similarly, let $E_{j}$ be the graph obtained from $E_{j-1}$ by splitting the vertex linked to two copies of $K_{r}$ into two vertices of degree $\frac{r}{2}$, say $u_{1}^{j}$ and $u_{2}^{j}$, then add an extra $K_{r}$ and link exactly $\frac{r}{2}$ edges from each $u_{i}^{j}$ to the new $K_{r}$ such that the resulting graph is $r$-regular as well, here $i=1,2$. The case for $r=4$ is shown in Figure 1.

It is obvious that $\gamma_{p}\left(E_{0}\right)=2$. Note that for any integer $k \geq 1, \gamma_{p}\left(E_{k}\right) \geq k+2$ since any power domination set of $E_{k}$ must contain at least one vertex in each added $K_{r}$ and also contain at least two vertices in the rest part isomorphic to the graph $E_{1}$ minus the added $K_{r}$. On the other hand, two vertices $a, u_{2}^{k}$ together with $k$ vertices, picking exactly one vertex from each added $K_{r}$ of $E_{k}$, form a power domination set of $E_{k}$. Since $\left|V\left(E_{k}\right)\right|=2 r+1+k(r+1), \gamma_{p}\left(E_{k}\right)=k+2=\frac{2 r+2+k(r+1)}{r+1}$.

The main result of the paper is as follows.

Theorem 2.2 Let $G$ be a connected claw-free 4-regular graph of order $n$. Then $\gamma_{P}(G) \leq \frac{n+1}{5}$ and the bound is sharp. 

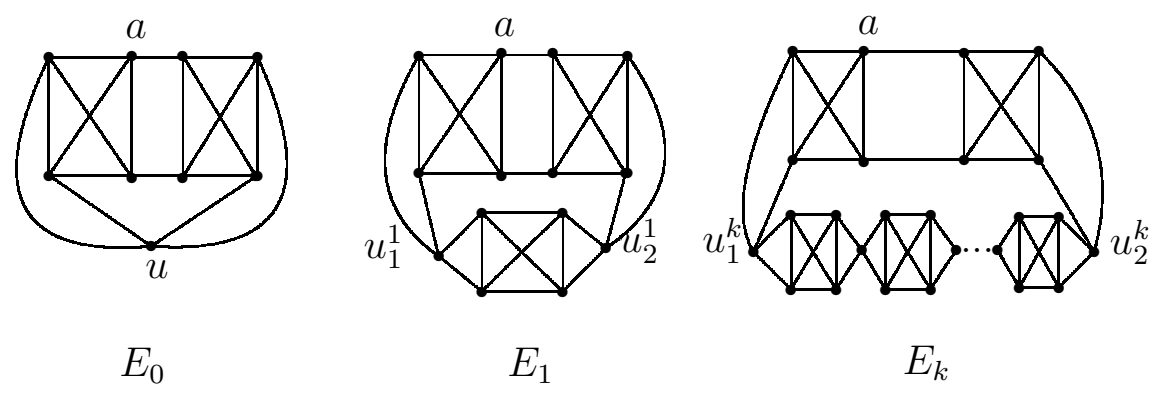

Figure 1. Counterexample graphs.

\subsection{Structure of the minimal counterexample $G$}

If the statement of Theorem 2.2 fails, then we suppose that $G$ is a counterexample with minimal $|V(G)|$. We have the following statement.

Lemma 2.1 $G$ is neither isomorphic to $K_{5}$ nor $I_{i}$ for $i \in\{1,2, \cdots, 8\}$.

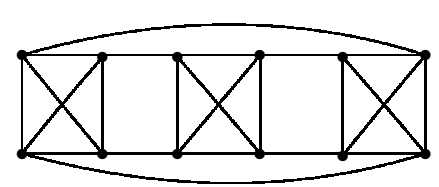

$I_{1}$

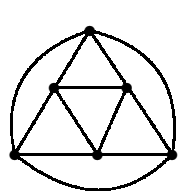

$I_{2}$
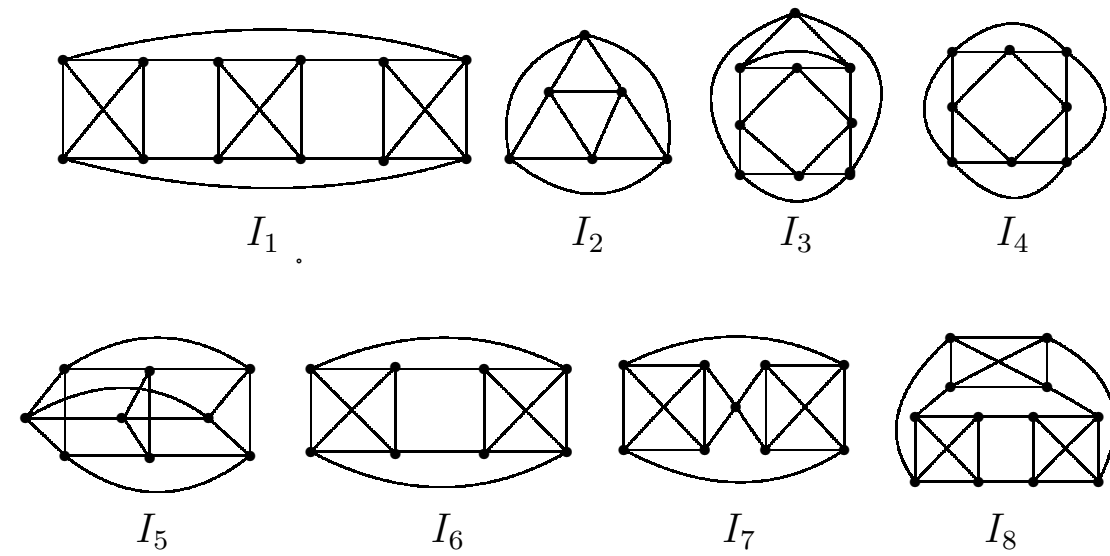

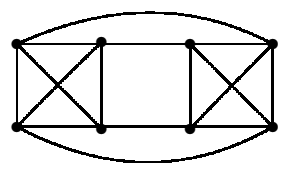

$I_{6}$

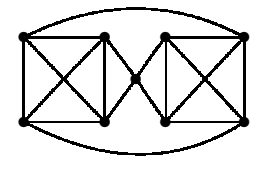

$I_{7}$

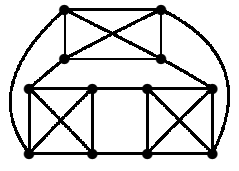

$I_{8}$

Figure 2. $I_{i}$ for $i \in\{1,2, \cdots, 8\}$.

Let $H$ be a subgraph of $G$. We say $v \in V(H)$ a saturated vertex of $H$ if $d_{H}(v)=d_{G}(v)=4$. If there is an induced cycle such that all of the vertices are saturated vertices of $H$, then we say the cycle a saturated cycle of $H$. Especially, a saturated triangle (or quadrilateral) is a saturated cycles of order three (or four). For convenience, if $G$ contains a subgraph isomorphic to $H$, then we only say that $G$ contains $H$.

For any integer $k \geq 2$, let $L_{k}$ be the graph obtained from $k$ disjoint copies of $K_{4}$ in linear order, say $D_{1}, D_{2}, \cdots, D_{k}$, by linking any two adjacent copies $\left(D_{i}, D_{i+1}\right)$ with two independent edges, where $i=1, \cdots, k-1$ (see Figure 3 ). 


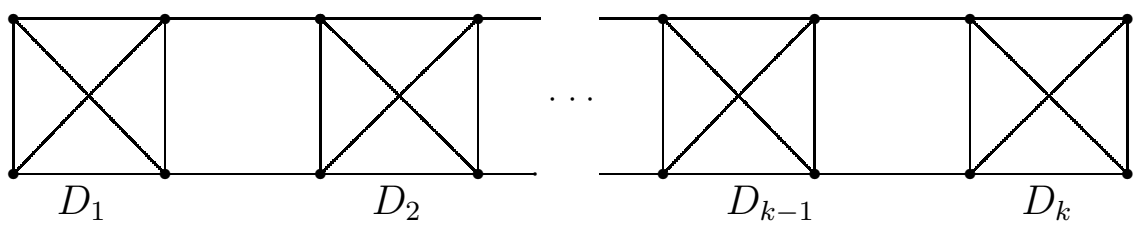

Figure 3. $L_{k}$ for some $k \geq 2$.

Lemma 2.2 $G$ does not contain $L_{k}$ for $k \geq 3$.

Proof. Otherwise, suppose that $G$ contains a subgraph $H$ isomorphic to $L_{3}$. Let $v_{1}, v_{2} \in V\left(D_{1}\right), v_{3}, v_{4} \in V\left(D_{3}\right)$ be the four vertices of degree three in $H$.

Suppose first that $H$ is an induced subgraph of $G$. Let $G^{\prime}$ be obtained from $G$ by deleting all saturated vertices in subgraph $H$ of $G$ and adding four extra edges $v_{1} v_{3}, v_{1} v_{4}, v_{2} v_{3}, v_{2} v_{4}$. Then $G^{\prime}$ is also a connected claw-free 4-regular graph of order $n^{\prime}$. Since $G^{\prime}$ is not a counterexample, $\gamma_{p}\left(G^{\prime}\right) \leq \frac{n^{\prime}+1}{5}$. Let $M^{\prime}$ be a $\gamma_{p}\left(G^{\prime}\right)$-set of $G^{\prime}$. If one of vertices $v_{1}, v_{2}, v_{3}$ and $v_{4}$ is in $M^{\prime}$, say $v_{1} \in M^{\prime}$, then $M=M^{\prime} \cup\{x\}$ is a PDS of $G$, where $x \in V\left(D_{3}\right) \backslash\left\{v_{3}, v_{4}\right\}$. Suppose now that $\left\{v_{1}, v_{2}, v_{3}, v_{4}\right\} \cap M^{\prime}=\emptyset$. Then $M^{\prime}$ together with one vertex of $D_{2}$ forms a PDS of $G$. Note that $\left|V\left(G^{\prime}\right)\right|=n-6$, then $\gamma_{p}(G) \leq|M| \leq \frac{n^{\prime}+1}{5}+1<\frac{n+1}{5}$, a contradiction.

Now suppose that $H$ is not an induced subgraph of $G$. If there are two extra edges linked four vertices $v_{1}, v_{2}, v_{3}$ and $v_{4}$, say $v_{1} v_{3}, v_{2} v_{4} \in E(G)$ then $G \cong I_{1}$. This contradicts Lemma 2.1. Then without loss of generality suppose that $v_{1} v_{3} \in E(G)$ but $v_{2} v_{4} \notin E(G)$. We can obtain $G^{\prime}$ from $G$ by exactly replacing the subgraph $H$ with a subgraph $H^{\prime}$ isomorphic to $K_{5}-e$ (the complete graph $K_{5}$ minus an edge) by identical their two vertices of degree three. Let $M^{\prime}$ be a $\gamma_{p}\left(G^{\prime}\right)$-set of $G^{\prime}$. It is easy to check that $V\left(H^{\prime}\right) \cap M^{\prime} \neq \emptyset$. Then $M=M^{\prime} \backslash V\left(H^{\prime}\right) \cup\left\{v_{2}, v_{4}\right\}$ is a PDS of $G$. Since $\left|V\left(G^{\prime}\right)\right|=n-7, \gamma_{p}(G) \leq|M| \leq \frac{n^{\prime}+1}{5}+1<\frac{n+1}{5}$, a contradiction.

Note that if $G$ contains a subgraph $L_{k}$ for some $k \geq 4$, then $G$ contains an induced subgraph isomorphic to $L_{3}$. Therefore $G$ does not contain any subgraph isomorphic to $L_{k}$ for $k \geq 3$.

Remark. From the above proof, we note that $G$ has some forbidden subgraphs, such as $L_{k}$ for $k \geq 3$. In fact, there are other forbidden subgraphs in $G$. That is, if $G$ contains such a subgraph, then we can replace the subgraph by some smaller subgraph to obtain a new graph $G^{\prime}$. There is a power domination set $S^{\prime}$ of $G^{\prime}$ such 
that $\left|S^{\prime}\right| \leq \frac{\left|V\left(G^{\prime}\right)\right|+1}{5}$. Based on $S^{\prime}$, we can get a desired power domination set of $G$ such that $\gamma_{p}(G) \leq \frac{|V(G)|+1}{5}$, contradicting that $G$ is a counterexample.
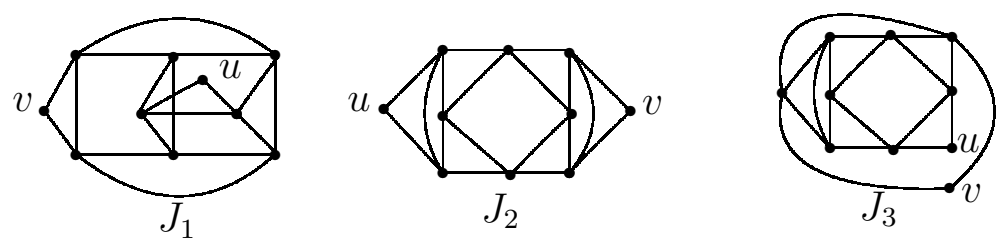

Operation $1 \Downarrow$
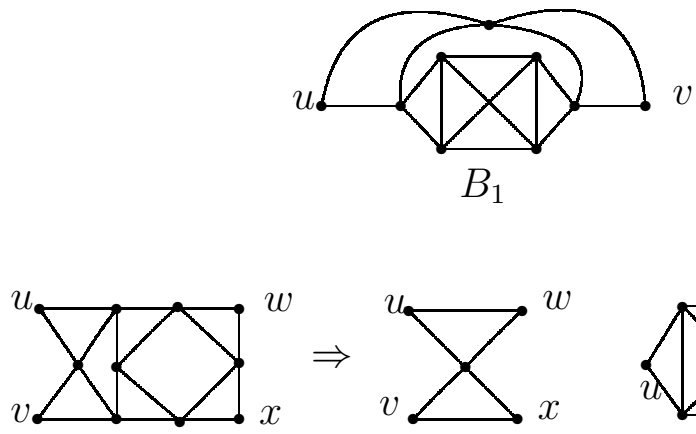

$J_{4}$

Operation 2

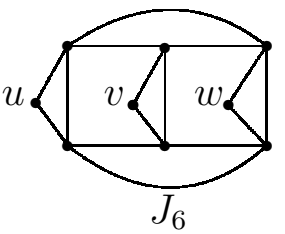

$\underbrace{w}_{J_{7}}=$

Operation 4
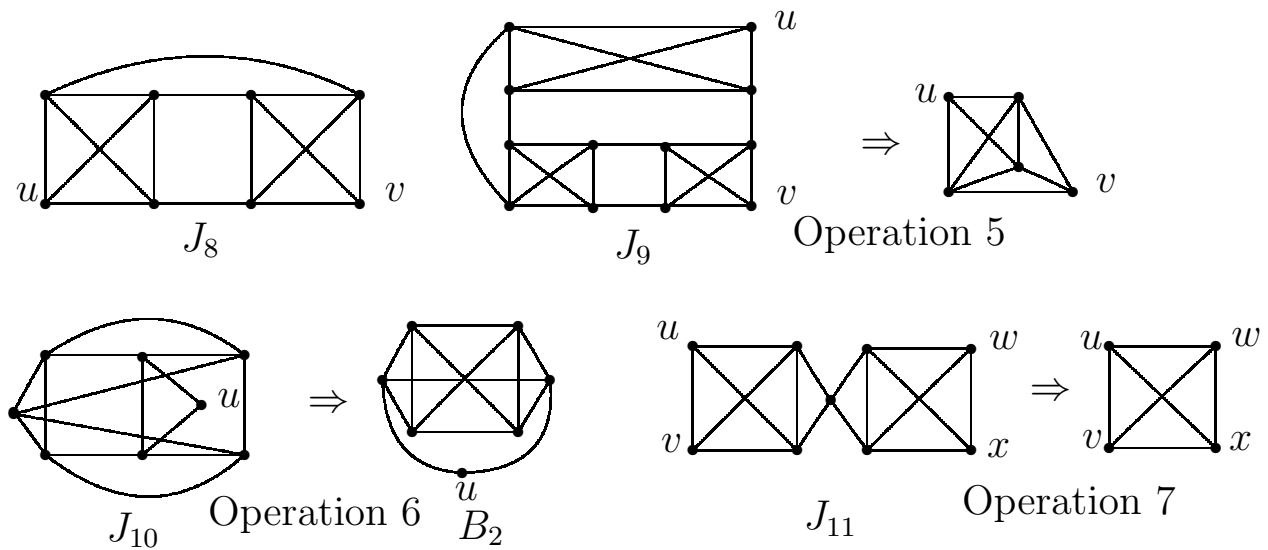


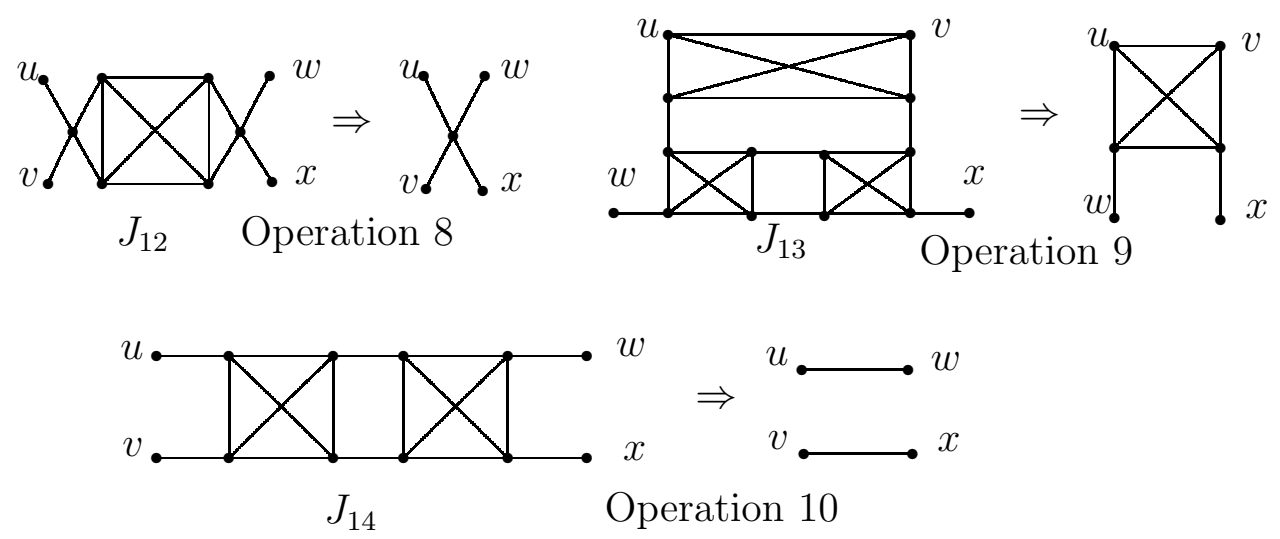

Figure 4. Forbidden subgraphs $J_{i}$ for $i \in\{1,2, \cdots, 14\}$.

Figure 4 give some replacement operations of graphs. For each operation, we replace $J_{i}$ by a smaller subgraph with identical labeled vertices. It is easy to check that the resulting graph is also 4-regular and claw-free. For example, for $i=1,2,3$, we can replace each of $J_{i}$ by $B_{1}$ by identifying the two vertices $u, v$ and keeping the neighborhood of the two vertices in $G^{\prime}=\left(V, E(G) \backslash E\left(J_{i}\right)\right)$.

Lemma 2.3 For each $i \in\{1,2, \cdots, 14\}$, we have the following statement.

(i) For $i \in\{1,2,3,4,5,8,9,10,12,13\}, G$ does not contain $J_{i}$ as a subgraph.

(ii) For $i \in\{6,7,11,14\}, G$ does not contain $J_{i}$ as an induced subgraph.

Proof. We only prove $G$ does not contain $J_{1}$ as a subgraph or $J_{14}$ as an induced subgraph. The proof for the other forbidden subgraphs is completely similar and we omit it here.

If $G$ contains $J_{1}$ as a subgraph, then we can obtain $G^{\prime}$ from $G$ by Operation 1. Then $\gamma_{p}\left(G^{\prime}\right) \leq \frac{n^{\prime}+1}{5}$, where $n^{\prime}$ is the order of $G^{\prime}$. Suppose that $M^{\prime}$ is a $\gamma_{p}\left(G^{\prime}\right)$-set. It is easy to check that $M^{\prime}$ contains at least one saturated vertex of $B_{1}$. If $M^{\prime}$ contains at least two saturated vertices of $B_{1}$, say $w_{1}, w_{2}$, then $M^{\prime} \backslash\left\{w_{1}, w_{2}\right\}$ together with any two saturated vertices of $V\left(J_{1}\right)$ will form a PDS of $G$. If $M^{\prime}$ contains exactly one saturated vertex of $B_{1}$, say $w_{1}$, then $w_{1}$ is in the copy of $K_{4}$ in $B_{1}$. Hence $u$ or $v$ must receive message from $M^{\prime} \backslash V\left(B_{1}\right)$. Therefore, $M^{\prime} \backslash\left\{w_{1}\right\}$ together with any saturated vertex nonadjacent to $u$ or $v$ in $J_{1}$ form a PDS of $G$. Thus, $|M| \leq\left|M^{\prime}\right| \leq \frac{n^{\prime}+1}{5} \leq \frac{n+1}{5}$. A contradiction.

If $G$ contains $J_{14}$ as an induced subgraph, then we can obtain $G^{\prime}$ from $G$ by operation 10. Suppose first that $G^{\prime}$ contains two components, where their order are 
$n_{1}$ and $n_{2}$, respectively. Let $M^{\prime}$ be a $\gamma_{p}\left(G^{\prime}\right)$-set. If $M^{\prime} \cap\{u, v, x, w\} \neq \emptyset$, say $u$, then we add a vertex $u^{\prime}$ with $d_{J_{14}}\left(u, u^{\prime}\right)=3$ to $M_{1} \cup M_{2}$. The resulting vertex set $M$ is a PDS of $G$. Then $|M| \leq \frac{n_{1}+1}{5}+\frac{n_{2}+1}{5}+1 \leq \frac{n-6+2}{5}+1 \leq \frac{n+1}{5}$, a contradiction. Suppose that $M^{\prime} \cap\{u, v, x, w\}=\emptyset$. By the symmetry if neither of $\{u, w\}$ can send message to each other, then $u$ receives message from $N_{G^{\prime}}(u) \backslash\{w\}$ which induces a copy of $K_{4}$ in $G^{\prime}$. Therefore $u$ can send message to $w$, a contradiction. Thus at least one vertex of $\{u, v, x, w\}$ can send message to its neighbor in $L_{2}$ of $G$, say $u$. $M=M^{\prime} \cup\left\{u^{\prime}\right\}$ is a PDS of $G$, where $d_{J_{14}}\left(u, u^{\prime}\right)=3$. Then $|M| \leq \frac{n_{1}+1}{5}+\frac{n_{2}+1}{5}+1 \leq \frac{n-6+2}{5}+1 \leq \frac{n+1}{5}$, a contradiction. If $G^{\prime}$ is the connected graph, then the discussion is similar and slightly easy than above. We omit it here.

Let $A=\left\{A_{1}, A_{2}, A_{3}\right\}$ be the collection of graphs in Figure 5. The three subgraphs need to pay our more attention.

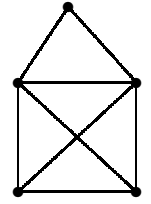

$A_{1}$

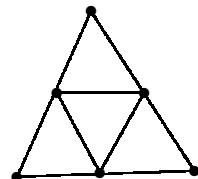

$A_{2}$

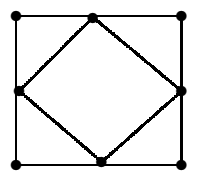

$A_{3}$

Figure 5. $A_{i}$ for $i \in\{1,2,3\}$.

For $i, j \in\{1,2,3\}$, let $A_{i} \circ A_{j}$ be the graph obtained from $A_{i}$ and $A_{j}$ by identifying exactly one vertex of degree two of them. We call the sharing vertex a focal vertex. Note that $A_{1} \circ A_{1} \cong J_{11}$. Then $G$ contains no induced subgraph isomorphic to $A_{1} \circ A_{1}$.

Lemma 2.4 Let $H_{1} \cong A_{i}$ and $H_{2} \cong A_{j}$ be two subgraphs of $G$, where $i \in\{1,2,3\}$ and $j \in\{2,3\}$. If there is a vertex $x$ in $H_{1} \cap H_{2}$, then $H_{1} \cup H_{2} \cong A_{i} \circ A_{j}$ and $x$ is $a$ focal vertex of $A_{i} \circ A_{j}$.

Proof. If $H_{1} \cong A_{1}$ and $H_{2} \cong A_{j}$ for $j \in\{2,3\}$, then the statement is obvious. Now we consider the case for $H_{1} \cong A_{i}$ and $H_{2} \cong A_{j}$, where $i, j \in\{2,3\}$. Let $C_{1}$ and $C_{2}$ be two saturated cycles of $H_{1}$ and $H_{2}$ respectively. We partition our proof into the following claims.

Claim 1. $\left|V\left(C_{1}\right) \cap V\left(C_{2}\right)\right| \leq 1$.

Proof. By the claw-freeness of $G$ and the configuration of $A_{2}$ and $A_{3}$, it is easy to check that $\left|V\left(C_{1}\right) \cap V\left(C_{2}\right)\right| \leq 2$. If the statement is false, then we suppose that $\left|V\left(C_{1}\right) \cap V\left(C_{2}\right)\right|=2$. 
If $C_{1}:=v_{1} v_{2} v_{3} v_{1}$ and $C_{2}:=u_{1} v_{2} v_{3} u_{1}$ are both saturated triangles, then by the configuration of $A_{2}$, there are two vertices $w_{1}$ and $w_{2}$ in $G$ such that all $w_{1} v_{1}, w_{1} v_{2}$, $w_{2} v_{1}$ and $w_{2} v_{3}$ are in $E(G)$. Since $C_{2}$ is the saturated triangle and by the 4-regular of $G, w_{1} u_{1}, w_{2} u_{1} \in E(G)$. By the claw-freeness of $G, w_{1} w_{2} \in E(G)$. Thus, $G \cong I_{2}$. This contradicts Lemma 2.1.

If $C_{1}:=v_{1} v_{2} v_{3} v_{1}$ is a saturated triangle and $C_{2}:=v_{1} v_{2} u_{1} u_{2} v_{1}$ is a saturated quadrilateral, then by the configuration of $A_{2}, u_{1} v_{3}, u_{2} v_{3} \in E(G)$. But it contradicts the configuration of $A_{3}$.

Finally we consider $C_{1}$ and $C_{2}$ are two saturated quadrilaterals. Note that now $C_{1}$ and $C_{2}$ share two adjacent vertices. Let $C_{1}:=w v v_{1} w^{\prime} w$ and $C_{2}:=w u u_{1} w^{\prime} w$. By the configuration of $A_{3}$, we have $u v, u_{1} v_{1} \in E(G)$. Then there are three vertices $w_{1}, w_{2}, w_{3}$ such that $w_{1} u, w_{1} u_{1}, w_{2} w, w_{2} w^{\prime}, w_{3} v, w_{3} v_{1}$ are in $E(G)$, here $w_{1}=w_{3}$ is allowed.

Suppose first $w_{1} \neq w_{3}$. Since $G$ is claw-free, $G\left[w_{1}, w_{2}, w_{3}\right]$ is either a clique or contains at most one edge. If $\left\{w_{1}, w_{2}, w_{3}\right\}$ induces a clique, then $G \cong I_{5}$. This contradicts Lemma 2.1. Now without loss of generality suppose $w_{1} w_{2} \in E(G)$. Since $G$ is claw-free and 4-regular, then $w_{1}$ and $w_{2}$ share a neighbor $w_{4}$. Now $G\left[V\left(H_{1} \cup\right.\right.$ $\left.\left.H_{2}\right) \cup\left\{w_{4}\right\}\right] \cong J_{1}$. This contradicts Lemma 2.3.

Now we consider $\left\{w_{1}, w_{2}, w_{3}\right\}$ is an independent set. Now $G\left[V\left(H_{1} \cup H_{2}\right)\right] \cong J_{6}$. This contradicts Lemma 2.3 as well.

If $w_{1}=w_{3}$, then $G\left[V\left(H_{1} \cup H_{2}\right)\right] \cong J_{10}$. A contradiction.

Claim 2. $\left|V\left(C_{1}\right) \cap V\left(C_{2}\right)\right|=0$.

Proof. Otherwise, suppose that there is a vertex $w \in V\left(C_{1} \cap C_{2}\right)$. Then we consider the three cases as follows.

Case 1. $C_{1}:=v_{1} v_{2} w v_{1}$ and $C_{2}:=u_{1} u_{2} w u_{1}$ are two saturated triangles.

By the configuration of $A_{2}$, let $u_{1} v_{1}, u_{2} v_{2} \in E(G)$ and there is another vertex $w_{1} \in V\left(H_{1}\right)$ adjacent to $v_{1}, v_{2}$. If $w_{1}$ is also the common neighbor of $u_{1}$ and $u_{2}$, then $G \cong I_{2}$, a contradiction.

Otherwise, there is the vertex $w_{2} \in V\left(H_{2}\right)$ (other than $w$ ) adjacent to both $u_{1}$ and $u_{2}$. Then $G\left[V\left(H_{1} \cup H_{2}\right)\right] \cong J_{5}$, a contradiction.

Case 2. $C_{1}:=u_{1} u_{2} w u_{1}$ is a saturated triangle and $C_{2}:=v_{1} v_{3} v_{2} w v_{1}$ is a saturated 
quadrilateral.

By the configuration of $A_{2}$ and $A_{3}$, without loss of generality say $u_{1} v_{1}, u_{2} v_{2} \in E(G)$ and there is a vertex $w_{1}\left(\neq v_{3}\right)$ adjacent to $u_{1}, u_{2}$. If $w_{1}$ is also the common neighbor of $v_{1}, v_{3}$ (or $\left.v_{2}, v_{3}\right)$, then $G\left[\left\{u_{1}, u_{2}, w\right\}\right]$ and $G\left[\left\{w_{1}, u_{1}, v_{1}\right\}\right]$ (or $G\left[\left\{w_{1}, u_{2}, v_{2}\right\}\right]$ ) are two saturated triangles with common vertex $u_{1}$ (or $u_{2}$ ). By the discussion of Case 1 above, we have $\gamma_{p}(G) \leq \frac{n+1}{5}$, a contradiction. Therefore there are three distinct vertices $w_{1}, w_{2}, w_{3}$ with all $w_{1} u_{1}, w_{1} u_{2}, w_{2} v_{2}, w_{2} v_{3}, w_{3} v_{3}, w_{3} v_{1}$ in $E(G)$. If $\left\{w_{1}, w_{2}, w_{3}\right\}$ induces a clique, then $G \cong I_{3}$. This contradicts Lemma 2.1.

Since $G$ is claw-free, $G\left[w_{1}, w_{2}, w_{3}\right]$ contains at most one edge. Suppose first that $w_{1} w_{2}$ or $w_{1} w_{3}$ is in $E(G)$. Then $w_{1}$ and $w_{2}$ (or $w_{3}$ ) share a common neighbor, say $w_{4}$. Hence $G\left[V\left(H_{1} \cup H_{2}\right) \cup\left\{w_{4}\right\}\right]$ contains a subgraph isomorphic to $J_{3}$. A contradiction. If $w_{2} w_{3} \in E(G)$, then $w_{2}$ and $w_{3}$ also share a common neighbor, say $w_{5}$. Now $G\left[V\left(H_{1} \cup H_{2}\right) \cup\left\{w_{5}\right\}\right]$ contains a subgraph isomorphic to $J_{2}$. A contradiction.

If $\left\{w_{1}, w_{2}, w_{3}\right\}$ is an independent set, then $G\left[V\left(H_{1} \cup H_{2}\right)\right] \cong J_{7}$. A contradiction.

Case 3. $C_{1}:=v_{1} v_{3} w v_{2} v_{1}$ and $C_{2}:=u_{1} u_{3} w u_{2} u_{1}$ are two saturated quadrilaterals.

Without loss of generality we can suppose that $u_{2} v_{2}, u_{3} v_{3} \in E(G)$. By the configuration of $A_{3}, v_{1}$ and $v_{3}$ share a neighbor $w_{1}, v_{1}$ and $v_{2}$ share a neighbor $w_{2}$. If $w_{1}$ is also the common neighbor of $u_{1}$ and $u_{3}$, then $G\left[\left\{w_{1}, u_{3}, v_{3}\right\}\right]$ is a saturated triangle of some $A_{2}$ intersecting the saturated quadrilateral $G\left[\left\{v_{1}, v_{2}, v_{3}, w\right\}\right]$. By the discussion of Case 2 above, we are done. If $w_{1}$ is the common neighbor of $u_{1}$ and $u_{2}$, then there is another saturated quadrilateral $G\left[\left\{w_{1}, v_{1}, v_{2}, u_{2}\right\}\right]$ intersecting $G\left[\left\{v_{1}, v_{2}, v_{3}, w\right\}\right]$ at $v_{1}$ and $v_{2}$. The case has been done in Claim 1 .

We suppose that there are two vertices $w_{3}, w_{4}$ with all of $w_{3} u_{1}, w_{3} u_{3}, w_{4} u_{1}, w_{4} u_{2}$ in $E(G)$. Base on the discussion above, we can suppose that all of $w_{1}, w_{2}, w_{3}$ and $w_{4}$ are distinct. By symmetry, if $w_{1} w_{2} \in E(G)$, then $G\left[\left\{w_{1}, w_{2}, v_{1}\right\}\right]$ is a saturated triangle intersecting $G\left[\left\{v_{1}, v_{2}, v_{3}, w\right\}\right]$ at $v_{1}$. By the discussion of Case 2 above, we are done. If $w_{3} w_{4} \in E(G)$, the similar discussion is valid as well.

Now we consider the case $w_{1} w_{2}, w_{3} w_{4} \notin E(G)$. Then $G\left[V\left(H_{1} \cup H_{2}\right)\right]$ contains a subgraph isomorphic to $J_{4}$. A contradiction.

Claim 3. $x \notin V\left(C_{1} \cup C_{2}\right)$.

Proof. Otherwise, without loss of generality say that $x \in V\left(C_{1}\right)$. By Claim 2, $C_{1}$ 
and $C_{2}$ are disjoint. First suppose that both $C_{1}$ and $C_{2}$ are saturated triangles. We can suppose that $C_{1}:=x v_{1} v_{2} x, C_{2}:=u_{1} u_{2} u_{3} u_{1}$ and $x u_{1}, x u_{2} \in E(G)$. Based on the configuration of $A_{2}$, all of $u_{1} v_{1}, u_{2} v_{2}, v_{1} u_{3}, v_{2} u_{3}$ are in $E(G)$. Then $G \cong I_{2}$. This contradicts Lemma 2.1.

Now suppose that $C_{1}:=x v_{1} v_{2} x$ is a saturated triangle and $C_{2}:=u_{1} u_{2} u_{3} u_{4} u_{1}$ is a saturated quadrilateral. Without loss of generality say $x u_{1}, x u_{2} \in E(G)$. Consider the configuration of $A_{2}$ and $A_{3}$, we have all of $u_{1} v_{1}, u_{2} v_{2} u_{3} v_{2}$ and $u_{4} v_{1}$ in $E(G)$. But this contradicts that $v_{1}$ and $v_{2}$ share a neighbor other than $x$.

Finally suppose that $C_{1}:=v_{1} x v_{2} v_{3} v_{1}$ and $C_{2}:=u_{1} u_{2} u_{3} u_{4} u_{1}$ are two saturated quadrilaterals. Without loss of generality say $u_{1} x, u_{2} x \in E(G)$. Based on the configuration of $A_{3}$, all of $u_{1} v_{1}, u_{2} v_{2}, v_{1} u_{3}$ and $v_{2} u_{4}$ are in $E(G)$. Since $G$ is claw-free, $v_{3} u_{3}, v_{3} u_{4} \in E(G)$. Therefore, $G \cong I_{4}$, contradicting Lemma 2.1.

From Claims 2 and 3, $x$ is exactly the focal vertex of $H_{1} \cup H_{2}$. This completes the proof.

Lemma 2.5 Let $H$ isomorphic to $A_{i}$ for $i=\{2,3\}$ be a subgraph of $G$, then $H$ is also an induced subgraph of $G$.

Proof. If $G$ contains a subgraph $H$ isomorphic to $A_{2}$, then let $C:=v_{1} v_{2} v_{3} v_{1}$ be the saturated cycle of $H$ and $w_{1}, w_{2}, w_{3}$ are three vertices of $H$ with $w_{i}$ is adjacent to both of $v_{i}$ and $v_{i+1}$, where $i \in\{1,2,3\}$ and the index of $v_{i}$ taken modulo 3 . If $w_{1} w_{2} \in E(G)$, then $w_{1}$ and $w_{2}$ share a neighbor other than $v_{2}$, say $w_{4}$. But now $G\left[V(H) \cup\left\{w_{4}\right\}\right]$ contains a subgraph isomorphic to $J_{5}$, a contradiction.

We now consider the case for $H$ isomorphic to $A_{3}$. Let $C:=u_{1} u_{2} u_{3} u_{4} u_{1}$ be the saturated cycle of $H$. For $i=1,2,3,4$, let $v_{i}$ be the vertex of degree 2 adjacent to both of $u_{i}$ and $u_{i+1}$ in $H$, here the index of $v_{i}$ are taken modulo 4 .

First suppose that there is an edge $v_{i} v_{i+1} \in E(G)$ for some $i \in\{1,2,3,4\}$, without loss of generality say $v_{1} v_{2}$.

If $v_{1}$ is adjacent to another vertex $w$ different from $v_{3}$ and $v_{4}$, then by the clawfreeness of $G$ and since $G$ is 4-regular, $w v_{2} \in E(H)$. Therefore $G[V(H) \cup\{w\}]$ contains a subgraph isomorphic to $J_{7}$, a contradiction. Similarly if $v_{1}$ is adjacent to $v_{4}$, we can obtain a contradiction as well. If $v_{1}$ is adjacent to $v_{3}$, then $v_{2} v_{3} \in E(H)$. 
Now $\left\{u_{1}, u_{2}, u_{3}, v_{1}, v_{2}, v_{3}\right\}$ induces a subgraph $H_{1}$ isomorphic to $A_{2}$. Note that the subgraph and $H$ share five common vertices. But this contradicts Lemma 2.4. If $v_{1} v_{4} \in E(G)$, then since $G$ is claw-free, $v_{3} v_{4}, v_{2} v_{3} \in E(H)$. Hence $G \cong I_{4}$. This contradicts Lemma 2.1 as well. It is similar to the case for $v_{1} v_{4}$ and $v_{2} v_{3}$ are edges of $H$.

Now none of $v_{i} v_{i+1}$ is an edge of $G$. If $v_{1} v_{3} \in E(G)$, then $v_{1}$ and $v_{3}$ share a neighbor, say $w$. Hence $\left\{v_{1}, u_{2}, u_{3}, v_{3}, u_{1}, v_{2}, u_{4}, w\right\}$ induces an $A_{3}$ with saturated cycle $C^{\prime}: v_{1} u_{2} u_{3} v_{3} v_{1}$. The cycle $C^{\prime}$ intersects $C$ at two vertices. This contradicts Lemma 2.4. Similarly, we have $v_{2} v_{4} \notin E(G)$. This means that $H$ is an induced subgraph of $G$.

This completes the proof.
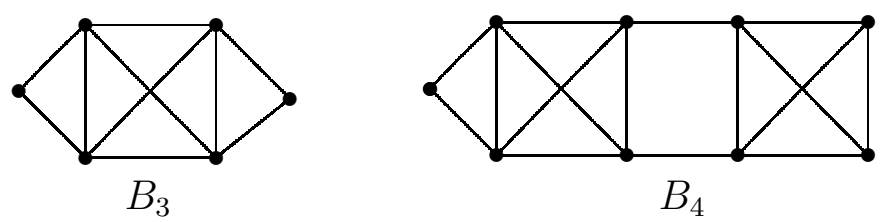

Figure 6. $B_{3}$ and $B_{4}$.

Let $B_{3}$ be the graph obtained from a $K_{5}$ by splitting its one vertex into two vertices of degree two. Let $B_{4}$ be the graph obtained from a $L_{2}$ by adding a new vertex and linking the vertex to two adjacent vertices of degree three in $L_{2}$, see Figure 6 . The following statement is easy to check and we omit its detailed proof.

Lemma 2.6 Let $H_{1}$ and $H_{2}$ be two subgraphs of $G$ sharing at least one vertex.

(i) If $H_{1} \cong A_{1}, H_{2} \cong A_{1}$, then $H_{1} \cup H_{2} \cong A_{1} \circ A_{1}, B_{3}$ or $K_{5}-e$.

(ii) If $H_{1} \cong A_{1}, H_{2} \cong L_{2}$, then $H_{1} \cup H_{2} \cong B_{4}$.

Note that $G \supsetneqq I_{7}$. If $G$ contains a induced subgraph $H$ isomorphic to $A_{1} \circ A_{1}$, then there is exactly one edge linking the two copies of $K_{4}$ of $H$.

Lemma 2.7 If $G$ contains a subgraph $H$ isomorphic to $B_{3}$, then $G$ contains a subgraph $H^{\prime}$ isomorphic to $B_{1}$ or $B_{2}$ (see Figure 4) such that $H \subset H^{\prime}$.

Proof. Let $u_{1}$ and $u_{2}$ be the two vertices of degree 2 in $H$. By the claw-freeness of $G$, if $u_{1} u_{2} \in E(G)$, then $u_{1}$ and $u_{2}$ share a common neighbor, say $w$, in $G$. Since $G$ is 4-regular and claw-free, $V(H) \cup\{w\}$ induces a $B_{2}$. 
Now suppose that $u_{1} u_{2} \notin E(G)$. If $u_{1}$ and $u_{2}$ have no common vertex, then $G\left[V(H) \cup N\left(u_{1}\right) \cup N\left(u_{2}\right)\right]$ contains a subgraph isomorphic to $J_{12}$. A contradiction.

Suppose that $u_{1}$ and $u_{2}$ have at least one common neighbor. Since $G$ is clawfree and 4-regular, $u_{1}$ and $u_{2}$ share exactly one common vertex, say $w$. (If $w^{\prime}$ is the common neighbor of $u_{1}$ and $u_{2}$ other than $w$, then $\left\{u_{1}, u_{2}, w\right\}$ together with the neighbor of $w$ (other than $w^{\prime}$ ) induces a claw). Let $w_{1}$ and $w_{2}$ be the fourth neighbors of $u_{1}$ and $u_{2}$, respectively. Note that $w_{1} w, w_{2} w \in E(G)$. The graph induced by $V(H) \cup\left\{w, w_{1}, w_{2}\right\}$ is isomorphic to $B_{1}$.

This completes the proof.

Lemma 2.8 If $G$ contains a subgraph $H$ isomorphic to $L_{2}$, then $G$ contains a subgraph $H^{\prime}$ isomorphic to $B_{4}$ such that $H \subset H^{\prime}$.

Proof. First show that $H$ is an induced subgraph of $G$. Let $V\left(D_{1}\right)=\left\{u_{1}, u_{2}, u_{3}, u_{4}\right\}$, $V\left(D_{2}\right)=\left\{v_{1}, v_{2}, v_{3}, v_{4}\right\}$ and $u_{1} v_{1}, u_{2} v_{2}$ be the two edges linked $D_{1}$ to $D_{2}$. If there are two edges linking $\left\{v_{3}, v_{4}\right\}$ to $\left\{u_{3}, u_{4}\right\}$, then $G \cong I_{6}$. This contradicts Lemma 2.1. If there is exactly one edge linking $\left\{v_{3}, v_{4}\right\}$ to $\left\{u_{3}, u_{4}\right\}$, say $u_{3} v_{3} \in E(G)$, then $G[V(H)] \cong J_{8}$, a contradiction.

Since $G$ is claw-free, $H$ is an induced subgraph of $G$ and $G$ is not isomorphic to $I_{7}$, any two nonadjacent vertices of degree three in $H$ have no common neighbor.

Suppose that statement fails, that is, $u_{3}$ and $u_{4}$ ( $v_{3}$ and $v_{4}$ respectively) have no common neighbor outside $H$. Let $w_{1}, w_{2}, w_{3}$ and $w_{4}$ are the four neighbors of $u_{3}, u_{4}, v_{3}$ and $v_{4}$ outside $H$, respectively. If $w_{1} w_{2} \in E(G)$ (or $w_{3} w_{4} \in E(G)$ ), then $w_{1}$ and $w_{2}$ (or $w_{3}$ and $w_{4}$ ) share two common neighbors outside $D_{1}$ (or $D_{2}$ ). Then $G$ contains a subgraph isomorphic to $L_{3}$, contradicting Lemma 2.2. So suppose that neither $w_{1} w_{2}$ nor $w_{3} w_{4}$ is in $E(G)$.

If one of $w_{1} w_{3}, w_{1} w_{4}, w_{2} w_{3}$ and $w_{3} w_{4}$, say $w_{1} w_{3}$, is in $E(G)$, then $w_{1}$ and $w_{3}$ have two common neighbors $w_{1}^{\prime}$ and $w_{3}^{\prime}$. $\left\{w_{1}, w_{3}, w_{1}^{\prime}, w_{3}^{\prime}\right\}$ induces a $K_{4}$, say $D_{3}$. If $\left\{w_{2}, w_{4}\right\} \cap\left\{w_{1}^{\prime}, w_{3}^{\prime}\right\} \neq \emptyset$, then either $G \cong I_{8}$ or $G\left[V(H) \cup\left\{w_{1}, w_{3}, w_{1}^{\prime}, w_{3}^{\prime}\right\}\right]$ is isomorphic to $J_{9}$. If $\left\{w_{2}, w_{4}\right\} \cap\left\{w_{1}^{\prime}, w_{3}^{\prime}\right\}=\emptyset$, then $G\left[V(H) \cup\left\{w_{1}, w_{2}, w_{3}, w_{4}, w_{1}^{\prime}, w_{3}^{\prime}\right\}\right]$ is isomorphic to $J_{13}$. For both cases, we get a contradiction. 
Now suppose that $W=\left\{w_{1}, w_{2}, w_{3}, w_{4}\right\}$ is an independent set. Then $G[V(H) \cup W]$ is isomorphic to $J_{14}$. This contradicts Lemma 2.3.

\subsection{The proof of Theorem 2.2}

In this section, we present a proof of our main result, namely, Theorem 2.2.

Let $T_{1}$ and $T_{2}$ be the graphs shown in Figure $7, F_{1}$ and $F_{2}$ be the graphs shown in Figure 8. For $i \in\{1,2\}$ and $j \in\{1,2,3\}$, let $T_{i} \circ A_{j}$ be the graph obtained from a $T_{i}$ and an $A_{j}$ by identifying a vertex of degree 2 in $T_{i}$ and a vertex of degree 2 in $A_{j}$. Since $G$ is a counterexample, it is easy to check that $G$ is not isomorphic to $T_{1} \circ T_{1}$.
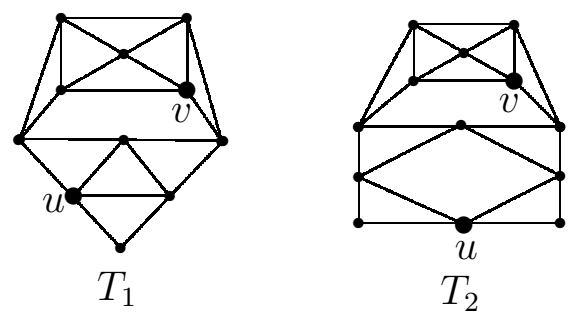

Figure 7. $T_{1}$ and $T_{2}$
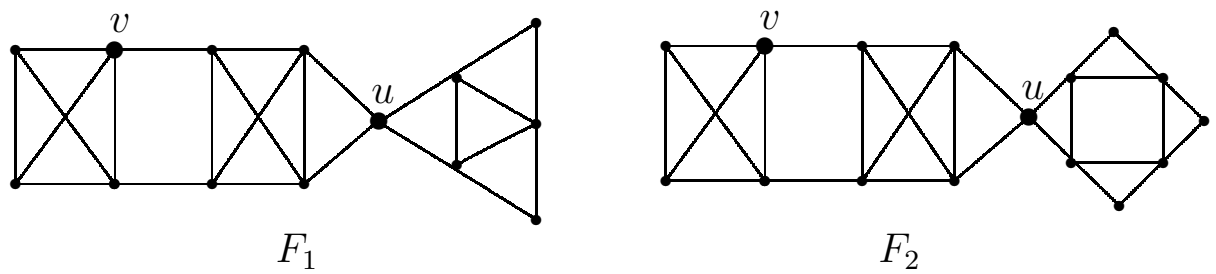

Figure 8. $F_{1}$ and $F_{2}$.

We give the following order to choose a packing $\mathcal{P}_{0}$ for $G$.

Initialize. $\mathcal{P}_{0}=\emptyset$.

Step 1. If $G$ contains $F_{i}$ for some $i=1,2$ and none saturated vertex of $F_{i}$ has message, then we add the focal vertex $u$ and a saturated vertex $v$ in $F_{i}$ with $d_{F_{i}}(u, v)=3$ to $\mathcal{P}_{0}$ (see, the two bigger vertices in Figure 8). Process Step 1 till $G$ contains no such an $F_{i}$. Then go to Step 2.

Step 2. If $G$ contains $T_{i} \circ A_{j}$ for some $i \in\{1,2\}, j \in\{1,2,3\}$ and none saturated vertex of $T_{i} \circ A_{j}$ has message, then we add the focal vertex (linked $T_{i}$ and $A_{j}$ ) $u$ and a saturated vertex $v$ with $d_{T_{i}}(u, v)=3$ to $\mathcal{P}_{0}$. Process Step 2 till $G$ contains no such a $T_{i} \circ A_{j}$. Then go to Step 3 . 
Step 3. If $G$ contains $T_{i}$ for some $i=1,2$ and none saturated vertex of $T_{i}$ has message, then we add two indictor vertices $u$ and $v$ with $d_{T_{i}}(u, v)=3$ to $\mathcal{P}_{0}$ (see, the two bigger vertices in Figure 7 ). Process the step till $G$ contains no such a $T_{i}$. Then go to Step 4.

Step 4. If $G$ contains $A_{i} \circ A_{j}$ for $i, j \in\{1,2,3\}$ and none saturated vertex of $A_{i} \circ A_{j}$ has message, then we add the focal vertex to $\mathcal{P}_{0}$. Process the step till $G$ contains no such an $A_{i} \circ A_{j}$. Then go to Step 5 .

Step 5. If $G$ contains $A_{i}$ for some $i \in\{1,2,3\}$ and none saturated vertex of $A_{i}$ has message, then we add one saturated vertex to $\mathcal{P}_{0}$. Process the step till $G$ contains no such an $A_{i}$.

Output. $\mathcal{P}_{0}$.

Remark. Notice that every vertex of $P_{0}$ is either a saturated vertex or a focal vertex in some subgraph of $G$, and before the vertex is chosen to $P_{0}$, it does not have message.

Lemma 2.9 Let $H$ be a subgraph isomorphic to some $A_{i}$ for $i=2,3$. Then $\mid V(H) \cap$ $\mathcal{P}_{0} \mid=1$.

Proof. Obviously $\left|V(H) \cap \mathcal{P}_{0}\right| \geq 1$. Suppose $x \in V(H) \cap \mathcal{P}_{0}$. By the remark above, at least one saturated vertex of $H$ has message. By Lemma 2.5, $H$ is an induced subgraph of $G$. By Lemma 2.4, if $H$ has a common vertex with other subgraph isomorphic to $A_{i}$ for $i \in\{1,2,3\}$, then the common vertex is the vertex of degree two of $H$. Therefore none vertices of $H$ is the saturated vertex in other subgraph isomorphic to $A_{i}$ for some $i$. According to the above choice order, we do not choose any vertex in $H$ but $x$. Hence $\left|V(H) \cap \mathcal{P}_{0}\right|=1$.

Lemma 2.10 Let $\mathcal{P}_{0}$ be the vertex subset of $G$ obtained by the choice order above. Then $\mathcal{P}_{0}$ is a packing of $G$.

Proof. Suppose the statement is false. That is, there are two vertices $x$ and $y$ of $\mathcal{P}_{0}$ such that $d(x, y) \leq 2$. In fact, we only need to consider the case for $d(x, y)=2$. We partition our discussion into two cases.

Case 1. $x$ (or $y$ ) is a focal vertex of some subgraph $H \cong A_{i} \circ A_{j}$ of $G$, where $i, j \in\{1,2,3\}$. 
Since $d(x, y)=2$, without loss of generality say that both of $x$ and $y$ are in $A_{i}$ of $H$. Now $\left|V(H) \cap \mathcal{P}_{0}\right| \geq 2$, by Lemma 2.9, we have $A_{i}=A_{1}$. Denote the subgraph isomorphic to $A_{1}$ in $H$ by $H_{1}$. Then the two vertices of degree three in $H_{1}$ are the saturated vertices in other copies of $A_{k}$ for some $k \in\{1,2,3\}$ or $B_{4}$. By Lemmas 2.3 and 2.6, there is a subgraph $H_{2}$ isomorphic to $B_{3}$ or $B_{4}$ such that $H_{1} \subset H_{2}$. If $y$ is chosen prior to $x$, then all the vertices of $A_{1}$ have message. It means that $x \notin P_{0}$, a contradiction. Now we suppose that $x$ is chosen prior to $y$.

If $H_{2} \cong B_{3}$, then by Lemma 2.7, there is a subgraph $H_{3}$ isomorphic to $B_{1}$ or $B_{2}$ such that $x, y \in V\left(H_{3}\right)$. Since $x \in V\left(A_{1} \circ A_{j}\right)$ and based on the configuration of $A_{1}$, $A_{j}$ is isomorphic to $A_{2}$ or $A_{3}$. By Lemma 2.5, $A_{j}$ is an induced subgraph of $G$. If $H_{3}$ is isomorphic to $B_{2}$, then one vertex of of degree two in $H_{2}$ is a saturated vertex of $A_{j}$. This contradicts Lemma 2.4. If $H_{3}$ is isomorphic to $B_{1}$, then $H_{2}$ and $A_{j}$ have two common vertices of degree two. By the configuration of $A_{2}$ or $A_{3}$, there is a subgraph $H_{4}$ isomorphic to $T_{1}$ or $T_{2}$ such that $x, y \in V\left(H_{4}\right)$.

Note that all the saturated vertices of $H_{4}$ have no message before $x, y$ are chosen. If there is a subgraph $H_{5}$ isomorphic to $F_{1}$ or $F_{2}$ such that $V\left(H_{4}\right) \cap V\left(H_{5}\right)$ isomorphic to $A_{2}$ or $A_{3}$, and none saturated vertices of $H_{5}$ has message before $x, y$ are chosen, then the focal vertex in $H_{5}$ which is also the vertex of degree two in $H_{4}$ should be chosen in Step 1. If there is no such an $H_{5}$, then we choose vertices of $H_{3}$ by Steps 2 or 3 . For both cases, we do not choose $x$ and $y$ in $\mathrm{H}_{2}$.

Now we consider that $H_{2} \cong B_{4}$. Note that $H$ and $H_{2}$ share an $A_{1}$. If $H \cong A_{1} \circ A_{1}$, then $H$ is also an induced subgraph of $G$, contradicting Lemma 2.3. If $H \cong A_{1} \circ A_{2}$ (or $H \cong A_{1} \circ A_{3}$ ), then both of $x$ and $y$ are in a subgraph $H_{3}$ isomorphic $F_{1}$ or $F_{2}$. Since $x$ is chosen prior to $y$, all the saturated vertices of $H_{3}$ have no message before $x$ and $y$ are chose. Then we do not choose $y$ in $H_{3}$, a contradiction.

Case 2. Neither $x$ nor $y$ is a focal vertex of any subgraph isomorphic to $A_{i} \circ A_{j}$ of $G$, where $i, j \in\{1,2,3\}$.

If $x$ is in some subgraph $H \cong F_{i}$ of $G$, where $i \in\{1,2\}$. If $y \in V(H)$, then $x$ is prior chosen to $y$. Note that $x$ and the focal vertex in $H$ are chosen to $P_{0}$ in Step 1 at the same time. Then $y \notin P_{0}$, a contradiction. Suppose that $y \notin V(H)$. Since $y$ is not the focal vertex, by Lemma 2.2, $y$ is a vertex of degree three in a copy of $A_{1}$, a 
contradiction.

If $x$ is in a copy of $A_{i}$ for some $i \in\{2,3\}$, then by Lemma2.9, $y$ is in another copy of $A_{j}$ for some $j \in\{1,2,3\}$. Since $d(x, y)=2$, both of $x$ and $y$ are in a subgraph $H$ isomorphic to $A_{i} \circ A_{j}$. Before $x, y$ being chosen, all the saturated vertices of $H$ have no message. If both of $x$ and $y$ are in some subgraph isomorphic to $T_{1}$ or $T_{2}$, then by the similar discussion as Case 1, we can get a contradiction. Otherwise, we choose the focal vertex other than $x$ or $y$ in $H$. A contradiction.

Now we suppose that $x$ is in the copy of $A_{1}$. Base on the above discussion, we only need to consider that $y$ is in another copy of $A_{1}$. If $x, y$ are in a subgraph $H$ isomorphic to $A_{1} \circ A_{1}$, then by Lemma 2.3, $G[V(H)] \cong I_{7}$, a contradiction. By Lemmas 2.6, we only need to consider that $x$ and $y$ are in a subgraph $H$ isomorphic to $B_{3}$ or $B_{4}$.

If $H \cong B_{3}$, then $y$ is the focal vertex, a contradiction. If $H \cong B_{4}$, then both of $x$ and $y$ are in a subgraph $H_{1}$ isomorphic to $F_{1}$ or $F_{2}$, where all the saturated vertices of $H_{1}$ have no message before $y$ being chosen. Note that $y$ is chosen prior to $x$. By Step 1, we do not choose $x$ in $H_{1}$, a contradiction.

This completes the proof.

Recall that for each integer $i \geq 0$ and a vertex set $S$ of $G, P^{0}(S)=S$ and $P^{i+1}(S)=\cup\left\{N_{G}[v]: v \in P^{i}(S)\right.$ such that $\left.\left|N_{G}[v] \backslash P^{i}(S)\right| \leq 1\right\}$. Note that if $H$ is isomorphic to $A_{i}$ for some $i \in\{1,2,3\}$, then from Step 5 of the choice order $P^{1}\left(\mathcal{P}_{0}\right)$ contains at least one saturated vertex of $H$.

Lemma 2.11 Let $G$ contain a subgraph $H$ isomorphic to $L_{2}$ and $\mathcal{P}_{0}$ be a packing denoted as above. Then $P^{1}\left(\mathcal{P}_{0}\right)$ contains at least one saturated vertex of $H$.

Proof. By Lemma 2.8, there is a subgraph $H_{1}$ isomorphic to $B_{4}$ such that $H \subset H_{1}$. If $H_{1}$ is contained in some subgraph isomorphic to $F_{1}$ or $F_{2}$, then by Step 1, all the saturated vertices of $H$ have message. Otherwise, by Step 5, at least one saturated vertices of $H$ could obtain message.

We extend the packing $\mathcal{P}_{0}$ of $G$ to a maximal packing and denote the resulting packing by $S_{0}$. 
Lemma 2.12 G has a sequence $S_{0} \subset S_{1} \subset \cdots \subset S_{l}$ such that the following holds:

(i) For all $0 \leq i \leq l-1,\left|S_{i+1}\right|=\left|S_{i}\right|+1$ and $\left|P^{\infty}\left(S_{i+1}\right)\right| \geq\left|P^{\infty}\left(S_{i}\right)\right|+5$.

(ii) $P^{\infty}\left(S_{l}\right)=V$.

Proof. Note that if $P^{\infty}\left(S_{0}\right)=V(G)$, then we are done. Now suppose that there is an $S_{i}$ for some $i \geq 0$ such that $M=P^{\infty}\left(S_{i}\right)$ does not contain all vertices of $G$. Denote $\bar{M}=V \backslash M$. Let $\mathcal{U}=\left\{u: u \in M, N_{G}(u) \backslash M \neq \emptyset\right\}$. It is obvious that $d_{M}(u) \geq 1$ and $2 \leq d_{\bar{M}}(u) \leq 3$ for each vertex $u \in \mathcal{U}$. We have the following statements.

Claim 1. For each $u \in \mathcal{U}, N_{G}(u) \backslash M$ induces a clique in $G$.

Proof. Suppose $x_{1}$ and $x_{2}$ are two neighbors of $u$ in $N_{G}(u) \backslash M$ and $u$ receives message from $v$ in $M$. Then $x_{1} v, x_{2} v \notin E(G)$. If $x_{1} x_{2} \notin E(G)$, then $\left\{u, x_{1}, x_{2}, v\right\}$ induces a claw. A contradiction.

Claim 2. For each vertex $x \in \bar{M}, d_{\bar{M}}(x) \geq 2$.

Proof. If all neighbors of $x$ are in $\bar{M}$, then we are done. Now suppose that $x$ has a neighbor $x^{\prime} \in M$. Note that $x^{\prime} \in \mathcal{U}$. Since $2 \leq d_{\bar{M}}\left(x^{\prime}\right) \leq 3$ and $N_{G}\left(x^{\prime}\right) \backslash M$ induces a clique, $d_{\bar{M}}(x) \geq 1$. If $d_{\bar{M}}(x)=1$, then let $x_{1}$ be the neighbor of $x$ in $\bar{M}$ and $N_{G}(x) \cap M=\left\{y_{1}, y_{2}, x^{\prime}\right\}$. Since none of vertices in $\left\{y_{1}, y_{2}, x^{\prime}\right\}$ sends message to $x$ and $N_{G}\left(y_{i}\right) \backslash M$ induces a clique for each $i \in\{1,2\}, N_{M}\left(x_{1}\right)=\left\{y_{1}, y_{2}, x^{\prime}\right\}$. Since $G$ is claw-free, $G\left[\left\{y_{1}, y_{2}, x^{\prime}\right\}\right]$ contains at least one edge. Therefore $G\left[\left\{x, x_{1}, y_{1}, y_{2}, x^{\prime}\right\}\right]$ contains an $A_{1}$ with two saturated vertices $x$ and $x_{1}$. Note that the two vertices have no message, contradicting to Step 5 of choice order.

Claim 3. Let $H \cong K_{4}$ be a subgraph of $G$ and $x$ be a vertex of $H$. If $x \in \bar{M}$ and there is a vertex $v$ such that $N_{H}(v)=\{x\}$, then $v \in \bar{M}$.

Proof. Since $G$ is claw-free and $N_{H}(v)=\{x\}, N_{G}[v] \backslash\{x\}$ induces a subgraph $H^{\prime}$ isomorphic to $K_{4}$. If $v \in M$, then $v$ will receive message from one of its neighbors in $H^{\prime}$. Since $H^{\prime} \cong K_{4}$, all vertices of $H^{\prime}$ have message. Now $v$ can send message to $x$, contradicting that $x \in \bar{M}$.

Note that $2 \leq d_{\bar{M}}(u) \leq 3$ for each vertex $u \in \mathcal{U}$. If there is a vertex $u \in \mathcal{U}$ such that $d_{\bar{M}}(u)=3$, then $u$ together with its three neighbors in $\bar{M}$, say $u_{1}, u_{2}, u_{3}$, induces a $K_{4}$. If two vertices of the three vertices, say $u_{1}$ and $u_{2}$, have a common vertex $u_{4}$ other than $u$ or $u_{3}$, then $\left\{u, u_{1}, u_{2}, u_{3}, u_{4}\right\}$ induces an $A_{1}$ with its saturated vertices $u_{1}$ and $u_{2}$. Note that neither $u_{1}$ nor $u_{2}$ has message. This contradicts Lemma 
2.11. Now suppose that each vertex in $\left\{u_{1}, u_{2}, u_{3}\right\}$ is adjacent exactly one vertex in $\bar{M} \backslash\left\{u_{1}, u_{2}, u_{3}\right\}$. Then let $S_{i+1}=S_{i} \cup\left\{u_{1}\right\}$. We have $\left|P^{\infty}\left(S_{i+1}\right)\right| \geq\left|P^{\infty}\left(S_{i}\right)\right|+5$.

Then we suppose that $d_{\bar{M}}(u)=2$ for each $u \in \mathcal{U}$. Let $u \in \mathcal{U}, N_{G}(u)=$ $\left\{v, v^{\prime}, u_{1}, u_{2}\right\}, v$ sends message to $u$ and $u_{1}, u_{2} \in \bar{M}$. We consider the two cases as follows.

Case 1. $v v^{\prime} \notin E(G)$.

By the claw-freeness of $G,\left\{u_{1}, u_{2}, v^{\prime}, u\right\}$ induces a $K_{4}$. If $u_{1}$ and $u_{2}$ share a neighbor other than $v^{\prime}, u$, say $w$, then $\left\{u_{1}, u_{2}, u, v^{\prime}, w\right\}$ induces an $A_{1}$ such that its two saturated vertices $u_{1}$ and $u_{2}$ have no message, a contradiction. By Claim $3, u_{1}$ and $u_{2}$ are adjacent to two distinct vertices $w_{1}$ and $w_{2}$ in $\bar{M} \backslash\left\{v^{\prime}, u, u_{1}, u_{2}\right\}$ respectively. If $w_{1} w_{2} \in E(G)$, then by the claw-freeness of $G$, both of $w_{1}$ and $w_{2}$ are in another copy of $K_{4}$. Then $\left\{u_{1}, u_{2}, w_{1}, w_{2}\right\}$ induces a saturated cycle in a copy of $L_{2}$, where none of saturated vertices of the $L_{2}$ has message, a contradiction. If $w_{1} w_{2} \notin E$, then by Claim 2, $w_{1}$ has a neighbor $w_{3} \in \bar{M}$ other than $u_{1}$. We define $S_{i+1}=S_{i} \cup\left\{w_{1}\right\}$. It is easy to check that $\left|P^{\infty}\left(S_{i+1}\right)\right| \geq\left|P^{\infty}\left(S_{i}\right)\right|+5$.

Case 2. $v v^{\prime} \in E(G)$.

Suppose that both of $u_{1}$ and $u_{2}$ are adjacent to $v^{\prime}$. Then whether $u_{1}$ and $u_{2}$ share a neighbor in $\bar{M}$ or not, by the similar discussion as Case 1 , we can obtain a contradiction or a desired $S_{i+1}$.

Now we suppose that neither $u_{1}$ nor $u_{2}$ is adjacent to $v^{\prime}$. If $u_{1}$ and $u_{2}$ share two neighbors other than $u$, then $G\left[N\left[u_{1}\right]\right] \cong A_{1}$ and none of two saturated vertices of $G\left[N\left[u_{1}\right]\right]$ has message, a contradiction. If $u_{1}$ and $u_{2}$ share exactly one neighbor other than $u$, say $w$, then let $w_{1}=N\left(u_{1}\right) \backslash\left\{w, u, u_{2}\right\}$ and $w_{2}=N\left(u_{2}\right) \backslash\left\{w, u, u_{1}\right\}$. Since $G$ is claw-free, $w_{1} w, w_{2} w \in E(G)$. Note that $w$ has no message (otherwise, $w$ receives message from $w_{1}$ or $w_{2}$, but it is impossible). Now $\left\{u, u_{1}, u_{2}, w, w_{1}, w_{2}\right\}$ induces an $A_{2}$ such that its saturated vertices $u_{1}, u_{2}, w$ have no message. A contradiction. If $u_{1}$ and $u_{2}$ do not have other common neighbors than $u$, then let $\left\{w_{1}, w_{2}\right\}=N\left(u_{1}\right) \backslash\left\{u, u_{2}\right\}$ and $\left\{w_{3}, w_{4}\right\}=N\left(u_{2}\right) \backslash\left\{u, u_{1}\right\}$. Since $G$ is claw-free, $w_{1} w_{2}, w_{3} w_{4} \in E(G)$. By Claim 2, we suppose without loss of generality that $w_{1}, w_{3} \in \bar{M}$. First suppose that $w_{2} \in \bar{M}$. If the two vertices of $N\left(w_{1}\right) \backslash\left\{w_{2}, u_{1}\right\}$ are also in $M$, then by Claim $1, N\left[w_{1}\right]$ induces an $A_{1}$ such that none of its saturated vertices $\left(w_{1}\right.$ and $\left.w_{2}\right)$ has message, a contradiction. 
Then at least one vertex of $N\left(w_{1}\right) \backslash\left\{w_{2}, u_{1}\right\}$ is in $\bar{M}$. Let $S_{i+1}=S_{i} \cup\left\{w_{1}\right\}$. We have $\left|P^{\infty}\left(S_{i+1}\right)\right| \geq\left|P^{\infty}\left(S_{i}\right)\right|+5$. The case for $w_{4} \in \bar{M}$ is similar completely. Now suppose that both of $w_{2}$ and $w_{4}$ are in $M$. By Claim 2, there is a vertex $t$ in $\bar{M}$ adjacent to $w_{1}$. If $t=w_{3}$, then by the claw-freeness of $G, w_{1}$ and $w_{3}$ share a neighbor, say $w_{5}$. Now $\left\{u, u_{1}, u_{2}, w_{1}, w_{2}, w_{3}, w_{4}, w_{5}\right\}$ induces an $A_{3}$. Since all vertices in the saturated cycle $u_{1} u_{2} w_{1} w_{3} u_{1}$ have no message, we get a contradiction. If $t \neq w_{3}$ then let $S_{i+1}=S_{i} \cup\left\{w_{1}\right\}$. Thus, $\left|P^{\infty}\left(S_{i+1}\right)\right| \geq\left|P^{\infty}\left(S_{i}\right)\right|+5$.

Now we consider the case for exactly one of $u_{1} v^{\prime}$ and $u_{2} v^{\prime}$ is in $E(G)$. Without loss of generality say $u_{1} v^{\prime} \in E(G)$ and $u_{2} v^{\prime} \notin E(G)$. By the front discussion, we can suppose that $d_{\bar{M}}\left(v^{\prime}\right)=2$ and $u_{3}$ is the other neighbor of $v^{\prime}$ in $\bar{M}$. By the claw-freeness of $G, u_{1} u_{3} \in E(G)$. Then $N[u] \cap\left\{u_{3}\right\}$ induces an $A_{2}$. Note that $u_{2} u_{3} \notin E(G)$ since each subgraph isomorphic to $A_{2}$ is also an induced subgraph of $G$. By Claim 2, we have $2 \leq d_{\bar{M}}\left(u_{2}\right) \leq 3$. If $d_{\bar{M}}\left(u_{2}\right)=3$, then let $S_{i+1}=S_{i} \cup\left\{u_{2}\right\}$. If $d_{\bar{M}}\left(u_{3}\right)=3$ then let $S_{i+1}=S_{i} \cup\left\{u_{3}\right\}$. For both cases, $\left|P^{\infty}\left(S_{i+1}\right)\right| \geq\left|P^{\infty}\left(S_{i}\right)\right|+5$. Now suppose that $d_{\bar{M}}\left(u_{2}\right)=d_{\bar{M}}\left(u_{3}\right)=2$. Let $w_{2}, w_{3} \in \bar{M}$ be another neighbors of $u_{2}$ and $u_{3}$ respectively. If $w_{2}=w_{3}$, then similar to the discussion in the paragraph above, we obtain a subgraph isomorphic to $A_{3}$ with all its saturated vertices have no message, a contradiction. If $w_{2} \neq w_{3}$, then let $S_{i+1}=S_{i} \cup\left\{u_{1}\right\}$. Thus, $\left|P^{\infty}\left(S_{i+1}\right)\right| \geq\left|P^{\infty}\left(S_{i}\right)\right|+5$.

Since $|V|$ is finite, there exists an integer $l$ such that $P^{\infty}\left(S_{l}\right)=V$.

We are now in a position to prove our main result, namely Theorem 2.2 . Proof. Let $G$ be a counterexample such that $|V(G)|$ is minimal. Let $S_{0}, S_{1}, \cdots, S_{l}$ be a desired sequence of Lemma 2.12. Then $\gamma_{P}(G) \leq\left|S_{l}\right|$. Since $S_{0}$ is a packing in $G$, we have $\left|P^{\infty}\left(S_{0}\right)\right|=\left|N\left[S_{0}\right]\right|=5\left|S_{0}\right|$. If $l=0$ then $n=5\left|S_{0}\right|$ and $\gamma_{p}(G) \leq\left|S_{0}\right| \leq \frac{n+1}{5}$. We are done. For $1 \leq i \leq l$, by Lemma $2.12(\mathrm{i})$, we have $\left|S_{i}\right|=\left|S_{i-1}\right|+1$. It means that $P^{\infty}\left(S_{i}\right) \geq P^{\infty}\left(S_{i-1}\right)+5$. Then $n=\left|P^{\infty}\left(S_{l}\right)\right| \geq\left|P^{0}\left(S_{0}\right)\right|+5 l=5\left(\left|S_{0}\right|+l\right) \geq 5 \gamma_{p}(G)$, Thus $\gamma_{p}(G) \leq n / 5$. It contradicts that $G$ is a counterexample. This completes the proof. 


\section{Linear-time algorithm for power domination in weighted trees}

The weighted domination problem has been well-studied in the last several decades. Farber [11], Natarajan and White [19] independently studied the classical domination in weighted trees. There is an extensive number of papers concerning the algorithmic complexity of the weighted domination problem in graphs, such as distance-hereditary graphs [24], chordal graphs [5], interval graphs [3, 20]. We refer to [4] for more results and details.

An natural extension of power domination is weighted power-domination. Let $G=$ $(V, E, w)$ be a weighted graph, where $w$ is a function from $V$ to positive real numbers.

Let $w(S)=\sum_{v \in S} w(v)$ be the weight of $S$ for any subset $S$ of $V$. The weighted power domination number, denoted by $\gamma_{p}^{w}(G)$, is defined as $\gamma_{p}^{w}(G)=\min \{w(S) \mid S$ is a power dominating set of $G\}$. The weighted power domination problem is to determine the weighted power domination number of any weighted graph.

Let $T=(V, E)$ be a tree with $n$ vertices. It is well known that the vertices of $T$ have an ordering $v_{1}, v_{2}, \cdots, v_{n}$ such that for each $1 \leq i \leq n-1, v_{i}$ is adjacent to exactly one $v_{j}$ with $j>i$. The ordering is call a tree ordering of the tree, where the only neighbor $v_{j}$ with $j>i$ is called the father of $v_{i}$ and $v_{i}$ is a child of $v_{j}$. For each $1 \leq i \leq n-1$, the father of $v_{i}$ is denoted by $F\left(v_{i}\right)=v_{j}$. For technical reasons, we assume that $F\left(v_{n}\right)=v_{n}$.

In this section, a linear time dynamic programming style algorithm is given to compute the exact value of weighted power domination number in any tree. This algorithm is constructed using the methodology of Wimer [22].

We make use of the fact that the class of rooted tree can be constructed recursively from copies of the single vertex $K_{1}$, using only one rule of composition, which combines two trees $\left(T_{1}, r_{1}\right)$ and $\left(T_{2}, r_{2}\right)$ by adding an edge between $r_{1}$ and $r_{2}$ and calling $r_{1}$ the root of the resulting larger tree $T$. We denote this as $\left(T, r_{1}\right)=\left(T_{1}, r_{1}\right) \circ\left(T_{2}, r_{2}\right)$.

In particular, if $D$ is a power dominating set of $T$, then $D$ splits two subsets $D_{1}$ and $D_{2}$ according to this decomposition. However, $D_{1}\left(D_{2}\right.$, respectively) may not be a power dominating set of $T_{1}$ ( $T_{2}$, respectively). We express this as follows: 
$(T, D)=\left(T_{1}, D_{1}\right) \circ\left(T_{2}, D_{2}\right)$. Let $T$ be a tree rooted at $r . \bar{T}$ is a new tree rooted at $r^{\prime}$, where $V(\bar{T})=V(T) \cup\left\{r^{\prime}\right\}$ and $E(\bar{T})=E(T) \cup\left\{r r^{\prime}\right\}$.

In order to construct an algorithm to compute weighted power domination number, we must characterize the possible tree-subset pairs $(T, D)$. For this problem there are five classes:

$[a]=\{(T, D) \mid D$ is a PDS of $T$ and $r \in D\}$;

$[b]=\{(T, D) \mid D$ is a PDS of $\bar{T}$ and $r \notin D\}$;

$[c]=\{(T, D) \mid D$ is a PDS of $T$, but not of $\bar{T}$ and $r \notin D\}$;

$[d]=\{(T, D) \mid D$ is a PDS of $T-r$, but it is not a PDS of $T$ and $r \notin D\}$;

$[e]=\{(T, D) \mid D$ is not a PDS of both $T$ and $T-r$, but all vertices of $T$ can be observed by $D$ if a message is given to $r$ in advance $\}$.

\begin{tabular}{c|ccccc}
$\circ$ & {$[a]$} & {$[b]$} & {$[c]$} & {$[d]$} & {$[e]$} \\
\hline$[a]$ & {$[a]$} & {$[a]$} & {$[a]$} & {$[a]$} & {$[a]$} \\
{$[b]$} & {$[b]$} & {$[b]$} & {$[b]$} & {$[c]$} & {$[c]$} \\
{$[c]$} & {$[c]$} & {$[c]$} & {$[c]$} & $\times$ & $\times$ \\
{$[d]$} & {$[b]$} & {$[b]$} & {$[d]$} & {$[e]$} & {$[e]$} \\
{$[e]$} & {$[c]$} & {$[c]$} & {$[e]$} & $\times$ & $\times$
\end{tabular}

Next, we must consider the expression $\left(T_{1}, D_{1}\right) \circ\left(T_{2}, D_{2}\right)$ with $\left(T_{1}, D_{1}\right)$ of class $[i]$ and $\left(T_{2}, D_{2}\right)$ of class $[j]$, where $i, j \in\{a, b, c, d, e\}$. The above table shows the results of $\left(T_{1}, D_{1}\right) \circ\left(T_{2}, D_{2}\right)$ in every possible cases. From this table, we obtain $[a]=[a] \circ[a] \cup[a] \circ[b] \cup[a] \circ[c] \cup[a] \circ[d] \cup[a] \circ[e] ;$

$[b]=[b] \circ[a] \cup[b] \circ[b] \cup[b] \circ[c] \cup[d] \circ[a] \cup[d] \circ[b] ;$

$[c]=[b] \circ[d] \cup[b] \circ[e] \cup[c] \circ[a] \cup[c] \circ[b] \cup[c] \circ[b] \cup[e] \circ[a] \cup[e] \circ[b] ;$

$[d]=[d] \circ[c]$

$[e]=[d] \circ[d] \cup[d] \circ[e] \cup[e] \circ[d]$.

The above formulation means that, for example, $(T, D)$ of class $[a]$ can be obtained from $\left(T_{1}, D_{1}\right)$ of class $[a]$ and $\left(T_{2}, D_{2}\right)$ of class $[a]$, or $\left(T_{1}, D_{1}\right)$ of class $[a]$ and $\left(T_{2}, D_{2}\right)$ of class $[b]$, or $\left(T_{1}, D_{1}\right)$ of class $[a]$ and $\left(T_{2}, D_{2}\right)$ of class $[c]$, or $\left(T_{1}, D_{1}\right)$ of class $[a]$ and $\left(T_{2}, D_{2}\right)$ of class $[d]$, or $\left(T_{1}, D_{1}\right)$ of class $[a]$ and $\left(T_{2}, D_{2}\right)$ of class $[e]$. It is easy to 
check that the above formulation is correct by inspection. The final step is to define the initial vector. In this case, for trees, the only basis graph is the tree with single vertex $v$. It is easy to obtain that the initial vector is $(w(v), \infty, \infty, 0, \infty)$, where ' $\infty$ ' means undefined. Now, we are ready to present the algorithm.

Algorithm WPDT. Compute the weighted power domination number for a weight tree.

Input: $\mathrm{A}$ tree $T=(V, E, w)$ with a tree ordering $v_{1}, v_{2}, \cdots, v_{n}$.

Output: The weighted power domination number $\gamma_{p}^{w}(T)$.

1. for $i:=1$ to $n$ do

2. initialize vector $[i, 1 . .5]$ to $\left[w\left(v_{i}\right), \infty, \infty, 0, \infty\right]$;

3. endfor

4. for $j:=1$ to $n-1$ do

5. $v_{k}=F\left(v_{j}\right)$;

6. vector $[k, 1]:=\operatorname{vector}[k, 1]+\min _{1 \leq m \leq 5} \operatorname{vector}[j, m]$;

7. vector $[k, 2]:=\min \left\{\operatorname{vector}[k, 2]+\min _{1 \leq m \leq 3} \operatorname{vector}[j, m]\right.$, vector $\left.[k, 4]+\min _{1 \leq m \leq 2} \operatorname{vector}[j, m]\right\}$;

8. $\operatorname{vector}[k, 3]:=\min \left\{\operatorname{vector}[k, 2]+\min _{4 \leq m \leq 5} \operatorname{vector}[j, m]\right.$, vector $[k, 3]+\min _{1 \leq m \leq 3} \operatorname{vector}[j, m]$, $\left.\operatorname{vector}[k, 5]+\min _{1 \leq m \leq 2} \operatorname{vector}[j, m]\right\}$;

9. $\operatorname{vector}[k, 4]:=\operatorname{vector}[k, 4]+\operatorname{vector}[j, 3]$;

10. $\operatorname{vector}[k, 5]:=\min \left\{\operatorname{vector}[k, 4]+\min _{4 \leq m \leq 5} \operatorname{vector}[j, m]\right.$, vector $\left.[k, 5]+\operatorname{vector}[j, 3]\right\}$.

11. endfor

12. $\gamma_{p}^{w}(T)=\min \{\operatorname{vector}[n, 1]$, vector $[n, 2], \operatorname{vector}[n, 3]\}$.

From the above argument, we can obtain the following theorem.

Theorem 3.1 Algorithm WPDT can output the weighted power domination number of any weighted tree $T=(V, E, w)$ in linear time $O(m+n)$, where $n=|V|$ and $m=|E|$.

\section{References}

[1] A. Aazami, M. D. Stilp, Approximation algorithms and hardness for domination with propagation, SIAM J. Discrete Math., 23:1382-1399, 2009. 
[2] T. L. Baldwin, L. Mili, M. B. Boisen Jr, R. Adapa, Power system observability with minimal phasor measurement placement, IEEE Trans. Power Systems, 8:707-715, 1993.

[3] A. A. Bertossi, A. Gori, Total domination and irredundance in weighted interval graphs, SIAM J. Discrete Math., 1:317-327, 1988.

[4] G. J. Chang, Algorithmic aspects of domination in graphs, in: D. Z. Du, P. M. Pardalos (Eds.), Handbook of Combinatorial Optimization, vol. 3, 1998, pp. 339-405.

[5] G. J. Chang, The weighted independent domination problem is NPcomplete for chordal graphs, Discrete Appl. Math., 143:351-352, 2004.

[6] G. J. Chang, P. Dorbec, M. Montassier, A. Raspaud, Generalized power domination of graphs, Discrete Appl. Math., 160:1691-1698, 2012.

[7] R. Diestel, Graph Theory, volume 173 of Graduate Texts in Mathematics, fourth ed., Springer, 2010.

[8] P. Dorbec, M.A. Henning, C. Löwenstein, M. Montassier, A. Raspaud, Generalized power domination in regular graphs, SIAM J. Discrete Math., 27: 1559-1574, 2013.

[9] P. Dorbec, M. Mollard, S. Klavžar, S. Špacapan, Power domination in product graphs, SIAM J. Discrete Math., 22: 554-567, 2008.

[10] M. Dorfling, M. A. Henning, A note on power domination in grid graphs, Discrete Appl. Math., 154:1023-1027, 2006.

[11] M. Farber, Domination and duality in weighted trees, Congr. Numer., 33:3-13, 1981.

[12] J. Guo, R. Niedermeier, D. Raible, Improved algorithms and complexity results for power domination in graphs, Algorithmica, 52:177-202, 2008.

[13] T. W. Haynes, S. M. Hedetniemi, S. T. Hedetniemi, M. A. Henning, Domination in graphs applied to electric power networks, SIAM J. Discrete Math., 15:519$529,2002$.

[14] T. W. Haynes, S. T. Hedetniemi, P. J. Slater, Fundamentals of domination in graphs, Marcel Dekker, New York, 1998. 
[15] T. W. Haynes, S. T. Hedetniemi, P. J. Slater, Domination in Graphs: Advanced Topics, Marcel Dekker, New York, 1998.

[16] J. Kneis, D. Molle, S. Richter, P. Rossmanith, Parameterized power domination complexity, Inform. Process. lett., 98:145-149, 2006.

[17] C. S. Liao, D. T. Lee, Power domination problem in graphs, Lecture Notes in Comput. Sci., 3595:818-828, 2005.

[18] L. Mili, T. Baldwin, A. Phadke, Phasor measurement placement for voltage and stability monitoring and control, in Proceedings of the EPRI-NSF Workshop on Application of Advanced Mathematics to Power Systems, San Francisco, CA, 1991.

[19] K. S. Natarajan, L. J. White, Optimum domination in weighted trees, Inform. Process. Lett., 7:261-265, 1978.

[20] G. Ramalingam, C. P. Rangan, Total domination in interval graphs revisited, Inform. Process. Lett., 27:17-21, 1988.

[21] C. Wang, L. Chen, C. Lu, Power domination in block graphs, J. Comb. Optim., 31:865-873, 2016.

[22] T. V. Wimer, Linear algorithms on $k$-terminal graphs, Ph.D. Thesis, Clemson University, 1987.

[23] G. Xu, L. Y. Kang, E. F. Shan, M. Zhao, Power domination in block graphs, Theoret. Comput. Sci., 359:299-305, 2006.

[24] H. G. Yeh, G. J. Chang, Weighted connected domination and Steiner trees in distance-hereditary graphs, Discrete Appl. Math., 87:245-253, 1998.

[25] M. Zhao, L. Y. Kang, G. J. Chang, Power domination in graphs, Discrete Math., 306:1812-1816, 2006. 